\title{
First principles prediction of exceptional mechanical and electronic behaviour of Titanite $\left(\mathrm{CaTiSiO}_{5}\right)$
}

\author{
Pratik Kr. Das ${ }^{\text {a,c,*, Sudip Kumar Mondal }}{ }^{b, c}$, Nibir Mandal ${ }^{c}$ \\ ${ }^{a}$ The Centre for Earth Evolution and Dynamics, University of Oslo, Oslo, N-0315, Norway \\ ${ }^{\mathrm{b}}$ Department of Physics, Jadavpur University, Kolkata 700032, India \\ ${ }^{\mathrm{c}}$ Faculty of Science, High Pressure and Temperature Laboratory, Jadavpur University, Kolkata 700032, India
}

\section{A R T I C L E I N F O}

\section{Key words:}

Negative elastic constant

Charge density

3D Young's modulus

Anisotropic optical properties

UV-shield material

DFT

\begin{abstract}
A B S T R A C T
Titanite $\left(\mathrm{CaTiSiO}_{5}\right)$ is a naturally occurring silicate phase, recently recognised as a potential material for immobilization of nuclear wastes, high-end ceramic lining and optical device development. The silicate undergoes a pressure-induced structural transition $\left(P 2_{1} / c\right.$ to $\left.C_{2} / c\right)$ at $\sim 3.5 \mathrm{GPa}$, which we confirm from density function perturbation theory. Using first principles calculations pressure-dependent structural parameters of both the phases are presented. This article features the mechanical property of $C 2 / c$ titanite characterized by a negative component $\left(C_{36}=-16.41 \mathrm{GPa}\right)$ of the elastic constant tensor $\left(C_{i j}\right)$, an enigmatic phenomenon seen not only in low symmetry (monoclinic), but also in the high symmetry system (cubic). We propose rotational kinematics of bonds, controlled by the valence charge distributions as a crucial atomic scale mechanism for structural collapse of the lattice under strain resulting in negative $C_{36}$. This kinematic model allows us to predict the necessary and sufficient condition for the pressure dependent softening of their shear elastic constants $\left(C_{44}\right.$ and $\left.C_{55}\right)$. The present study also sheds a new light upon the electronic properties of titanite, accounting for the intraband and interband transitions that influence the optical activity. Its anisotropic optical properties of titanite evaluated in the range 0-60 eV are marked by an attractive optical behaviour of this phase. It is transparent in the visible spectra, but showing excellent absorption and reflectivity in the UV region. We thus project titanite as an industrially potential UV shield material. Our theoretical estimate yields the highest value of anisotropic refractive index, 2.21 in [001].
\end{abstract}

\section{Introduction}

Titanite is a multifunctional nesosilicate phase [1], well known as a versatile host for rare earth elements (REE) [2-5], and also widely used as U-Pb geochronometer for dating geological events [6]. This crystalline phase, rich in $\mathrm{TiO}_{2}$ content, is a demanding material owing to its applications for developing strategic matrices for nuclear waste disposal, which is currently a challenging and intriguing field of research $[2,4]$. Various properties of single-crystal titanite have been investigated in ambient and high-pressure environments with high-end laboratory techniques, such as DAC (diamond anvil cell) and multi anvil experiments [7-13]. Salje et al. [14] showed the softening of shear modulus through annealing in a radiation damaged titanite sample. Their estimate of the bulk modulus is found to be much lower than that of Angel et al. [15]. However, the literature lacks any reliable experimental or theoretical data for the $2^{\text {nd }}$ order elastic constants of titanite, which are the pre-requisite mechanical parameters in order to explore the viability of this crystalline phase in extreme industrial applications. Our present study provides an account of the structural morphology and the physical properties of titanite phases $\left(P 2_{1} / c\right.$ and $\left.C 2 / c\right)$ at elevated pressure, and offers an insight into the structural dependence of their elastic properties. The structural analysis evaluates the relative rotation of different atomic bonds and subsequent changes in the alignment of their corresponding polyhedra under imposed strains.

A large part of our present article focuses upon the elasticity of titanite, as it is a remarkable macroscopic mechanical property of solids, extensively used to study a range of atomic scale phenomena, such as lattice instability, spin transitions, lattice dynamics and phonon instability [16-18]. An enormous volume of the existing literature deals with the elasticity of crystalline phases, but mostly as positive quantities [19-23]. Despite a number of existing continuum models [24-27], the elasticity as a negative quantity is still an enigma because the underlying atomic scale physics for such an unusual mechanical behaviour has remained unexplored. Experimental studies suggest that crystalline materials of low as well as high symmetry structures may have negative elastic constants, implying that the negative elasticity is not a direct consequence

\footnotetext{
* Corresponding author at: The Centre for Earth Evolution and Dynamics, University of Oslo, Oslo, N-0315, Norway.

E-mail address: pratik@geo.uio.no (P.Kr. Das).
} 


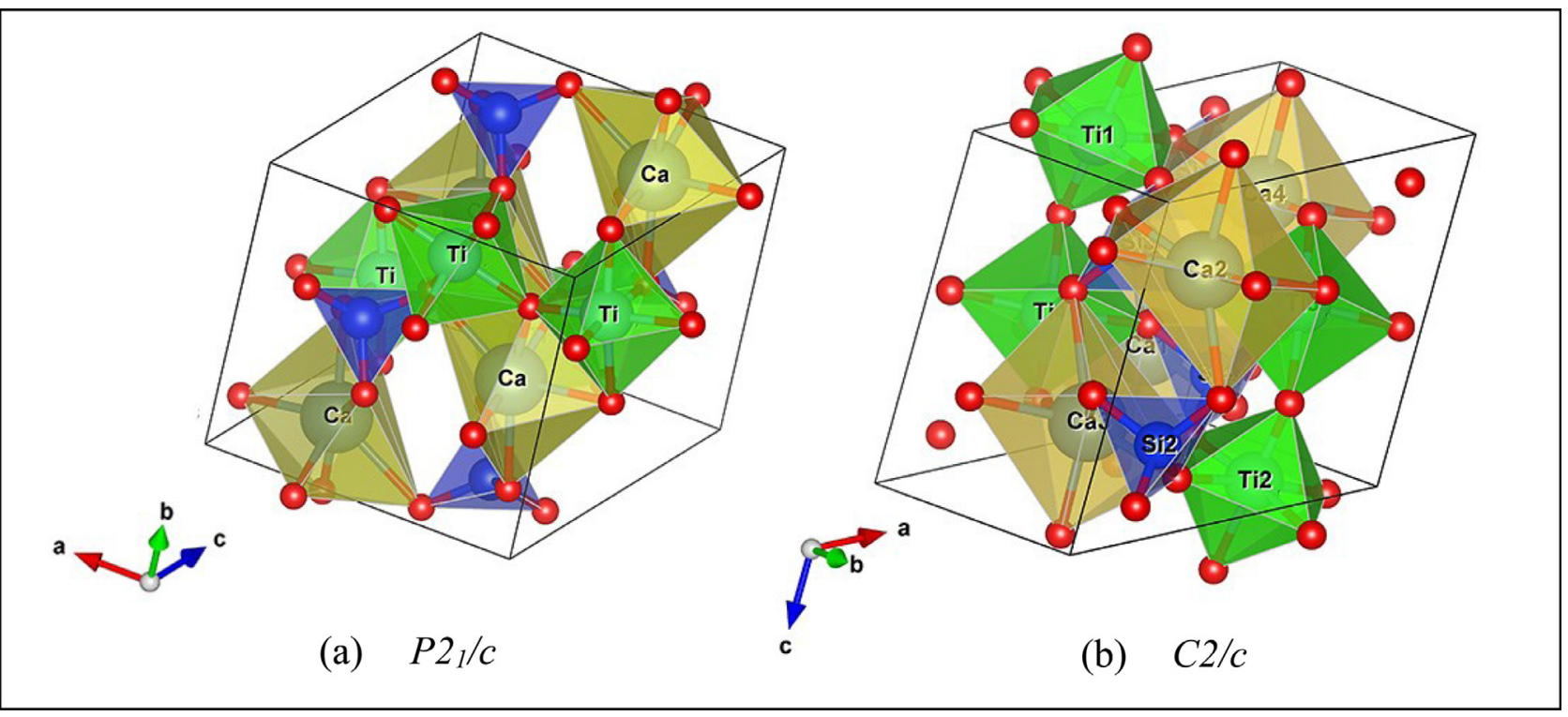

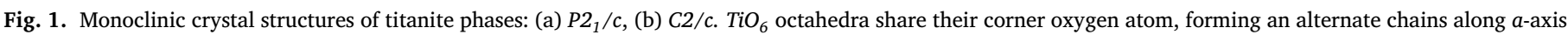
in $\mathrm{P}_{1} / \mathrm{c}$, whereas along the $c$ axis in $\mathrm{C} 2 / \mathrm{c}$. The two sides of a $\mathrm{TiO}_{6}$ octahedron are shared by edges of two distorted $\mathrm{CaO}_{7}$ polyhedra.

of the crystal symmetry [28,29]. Although the reports on the negative elastic constants are scanty, experimental investigations performed on high symmetry cubic phases confirm the existence of this exceptional mechanical behaviour of solids [28-31]. Our first principles calculations predict the negative component $\left(C_{36}\right)$ of the elastic constant tensor $\left(C_{i j}\right)$ of $C 2 / c$ titanite, which, to the best of our knowledge, is reported for the first time. We provide an atomic level basis of such negative elastic behaviour, taking into account the rotational bond kinematics under a given strain to the crystal.

A recent study by Malcherek and Fischer [32] has compared the phonon dispersions of $P 2_{1} / c$ and $C 2 / c$ phases of titanite $\left(\mathrm{CaTiSiO}_{5}\right)$ and mayalite $\left(\mathrm{CaSnSiO}_{5}\right)$, showing negative acoustic branches in $\mathrm{C} 2 / \mathrm{c}$ titanite phase along the direction [001]. They have proposed that $P 2_{1} / c$ titanite can be obtained from $C 2 / c$ phase by destabilizing a continuous phonon mode along [001] by lowering the temperature. However, high pressure behaviour of $P 2_{1} / c$ titanite is still absent in the literature. Secondly, despite a great demand of titanite for its electronic and optical properties in materials engineering, in-depth theoretical and experimental studies to assess these properties are still awaited. A line of our present study aims to meet this gap. We predict titanite as a semiconductor with an electronic band gap of $3.2 \mathrm{eV}$. We are also motivated to explore the anisotropic optical behaviour of this silicate phase in a frequency range 0-60 eV. Interestingly, our findings provide a new insight into the applicability of titanite as UV-shield materials.

\section{Computational method}

Our calculations were performed within the framework of DFT using the VASP5.3 [33]. PAW potentials [34] provided with VASP explicitly treat [3p4s], [3d4s], [3s3p] and [2s2p] orbitals for $\mathrm{Ca}, \mathrm{Ti}, \mathrm{Si}$ and $O$ as valence states with the core radii 1.746, 1.323, 1.312 and $0.82 \AA$, respectively. We considered the revised GGA (RPBE) scheme for the exchange-correlation effects [35]. To account for the electron correlation of Ti $d$ electrons we performed GGA+U (on-site correction for Coloumb interaction) calculations by incorporating the Hubbard-type term in the density functional following the method proposed by Dudarev et al. [36]. Coulomb repulsion is thus considered explicitly using $U_{\text {effective }}=U-J$, where $J=0$. U is evaluated self consistently by varying its values in the range 2 to $8 \mathrm{eV}$ where $\mathrm{U}=4 \mathrm{eV}$ yields an optimum value of band gap. All the simulations were carried out on 32 atom cell for both the titanite phases. We fixed the kinetic energy cut-off at 1000
eV. The conjugate gradient algorithm was employed to execute geometrical optimization in finding the ground state electronic structure under the strict electronic and ionic convergence criteria of $10^{-6} \mathrm{eV}$ and $10^{-3}$ $\mathrm{eV} /$ atom, respectively. The brillouin zones were sampled by $3 \times 2 \times 3$ and $4 \times 4 \times 3$ Monkhorst-Pack [37,38] k-point grid, which gave rise to 10 and 21 irreducible k-points in the brillouin zone. We used Phonopy [39] for lattice dynamical calculation with $2 \times 2 \times 2$ supercell. The force constants were calculated using DFPT without constraining their symmetry and they were interpolated using $17 \times 17 \times 17$ q-mesh for the full dispersion curve. Such dense q-mesh was chosen to increase the accuracy of the phonon dispersion.

\section{Results and discussions}

\subsection{Structural analysis}

Titanite $\left(\mathrm{CaTiSiO}_{5}\right)$ crystallizes with monoclinic symmetry (space group: $P 2_{1} / c$; No. 14) (Fig. 1 a) at ambient condition [9,40,41]. This phase undergoes structural transitions with increasing pressure as well as temperature. Under ambient pressure it transforms into another monoclinic phase with space group $A 2 / a$ at a temperature of $\sim 500 \mathrm{~K}[11,41-$ 43]. Kunz et al. [44] predicted the same transition with pressure at 6.9 GPa from DAC experiments. Some workers have reported another phase transition of titanite, i.e., $P 2_{1} / c$ to $C 2 / c$ (Fig. 1 b) phase at 487 $\mathrm{K}[11,12,42]$. This transition is reported to occur also at a hydrostatic pressure of $\sim 3.5 \mathrm{GPa}[10]$, and it is inferred to be analogous to the 825 $\mathrm{K}$ transition by Kunz et al. [45] from a powder diffraction study. Using lattice dynamics we predict $P 2{ }_{1} / c$ to $C 2 / c$ transition to occur within $5 \mathrm{GPa}$. Fig. 2 shows the phonon dispersion curves for $P 2_{1} / c$ phase at 0 and $5 \mathrm{GPa}$ along the high symmetry points G-Z-B-D-G-A. The optical modes shift towards higher frequency regions, whereas the acoustic modes soften to some negative values with increasing pressure. The phonon dispersion at $0 \mathrm{GPa}$ suggests that the $P 2_{1} / c$ phase is dynamically stable, but it develops a negative acoustic branch along G-A at $5 \mathrm{GPa}$, implying that the $P 2_{1} / c$ phase becomes dynamically unstable at $5 \mathrm{GPa}$. The phonon dispersion analysis is in agreement with previous finding of $P 2_{1} / c$ to $C 2 / c$ transition at $\sim 3.5 \mathrm{GPa}$ [10].

$\mathrm{Ca}, \mathrm{Ti}, \mathrm{Si}$ and $\mathrm{O}$ atoms occupy the $4 e$ wyckoff sites in the lattice structure of the monoclinic phase $\left(P 2_{1} / c\right)$. Si atoms occur in a four-fold coordination with $\mathrm{O}$ atoms to constitute $\mathrm{SiO}_{4}$ tetrahedra, which act as a building block of the titanite structure. Ti and $\mathrm{Ca}$ atoms occur in six- and 


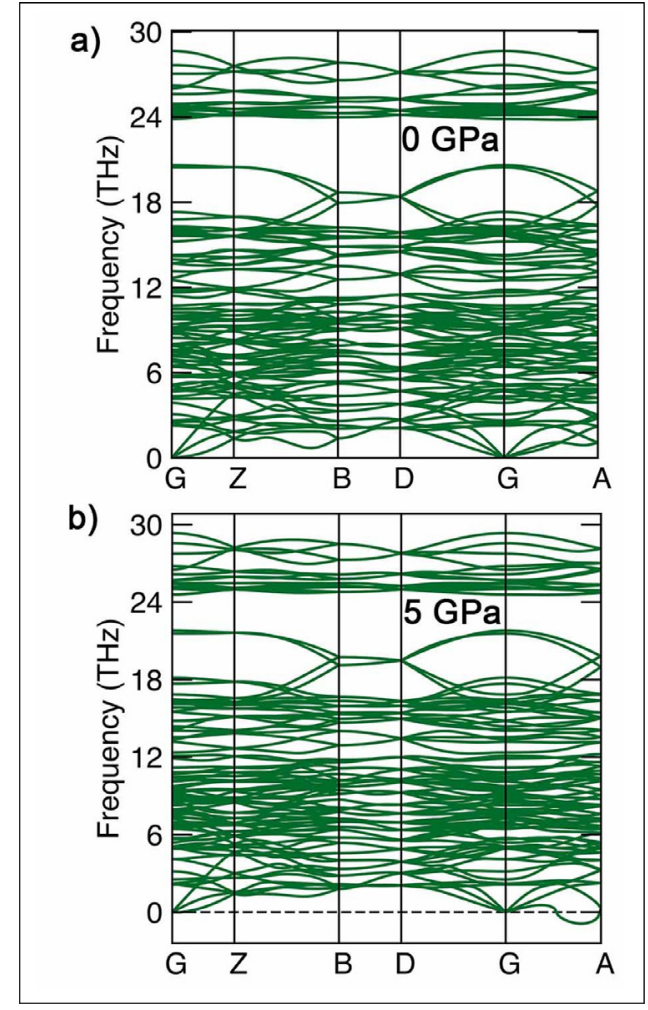

Fig. 2. Phonon dispersion curves for $P 2_{1} / c$ titanite phase at (a) $0 \mathrm{GPa}$ and (b) $5 \mathrm{GPa}$.

seven-fold coordination with $\mathrm{O}$ atoms, where the $\mathrm{TiO}_{6}$ octahedra form corner-linked chains along the $a$ axis (Fig. 1a). These octahedra are tilted in opposite directions alternately along the chains, sharing their edges with $\mathrm{CaO}_{7}$ polyhedra arranged in chains parallel to [101]. $\mathrm{SiO}_{4}$ tetrahedra share their corners with both $\mathrm{TiO}_{6}$ and $\mathrm{CaO}_{7}$ polyhedra $[12,41,46]$. A detailed description of the crystal structure can be found in Speer and Gibbs' work [41]. The Ti atoms are slightly off-centred in the polyhedra arranged along the $a$ axis at room temperature, which results in an anti-ferroelectric distortion pattern, but without any net inherent ferroelectric moment. However, the tetrahedral geometry remains unaffected even in the presence of any external electric field applied along $a$ direction. Zhang et al. predicted that switching of Ti displacements is not possible in strong electric fields (at least $35 \mathrm{kV} / \mathrm{cm}$ ) along the $a$ direction in a temperature range of 0 to $500 \mathrm{~K}$ [11].

Table 1 presents the pressure dependent variations of the structural parameters of both titanite phases $\left(P 2_{1} / c\right.$ and $\left.C 2 / c\right)$ in the pressure $(p)$ range 0 to $5 \mathrm{GPa}$. The cell volume of $P 2_{1} / c$ phase reduces by $\sim 4.53 \%$ at $p=5 \mathrm{GPa}$, whereas the lattice parameters, $a, b$ and $c$, reduce by $\sim 2.15 \%$, $0.77 \%, 2.19 \%$ respectively, implying that the $P 2_{1} / c$ phase has the lowest compressibility along the $b$ axis. The $C 2 / c$ phase shows similar pressure dependent changes in $a, b$ and $c(\sim 2.17 \%, 0.81 \%$ and $1.64 \%$, respectively). However, the $c$ axis of $C 2 / c$ phase shows remarkably higher incompressibility than the $P 2_{1} / c$ phase. We also evaluated polyhedral volumes as a function of $p . \mathrm{CaO}_{7}$ polyhedra undergo large modifications of their volumes with increasing $p$. In contrast, $\mathrm{SiO}_{4}$ tetrahedral volumes show much smaller pressure-induced modifications for both the phases. In case of $\mathrm{P} 2_{1} / c$ phase, $\mathrm{Ca}-\mathrm{O}$ bond lengths $\left(\mathrm{CaO}_{7}\right.$ polyhedra) reduce by $\sim 1.97 \%(\sim 10.06 \%)$ at $p=5 \mathrm{GPa}$, which is significantly low in the $C 2 / c$ phase, $\sim 0.27 \%(\sim 6.34 \%)$.

\subsection{Elastic constant tensor}

We have calculated the second order elastic constant tensors $\left(C_{i j}\right)$ of single crystals for both the titanite phases $\left(P 2_{1} / c\right.$ and $\left.C 2 / c\right)$ under hy-

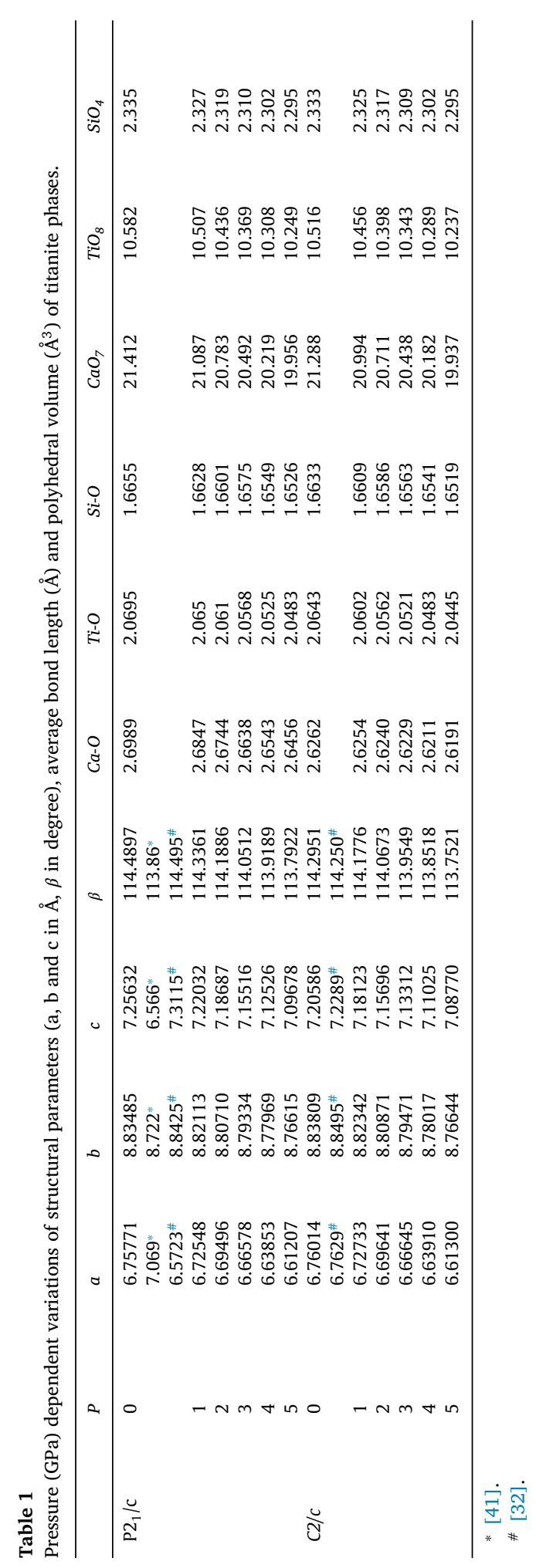




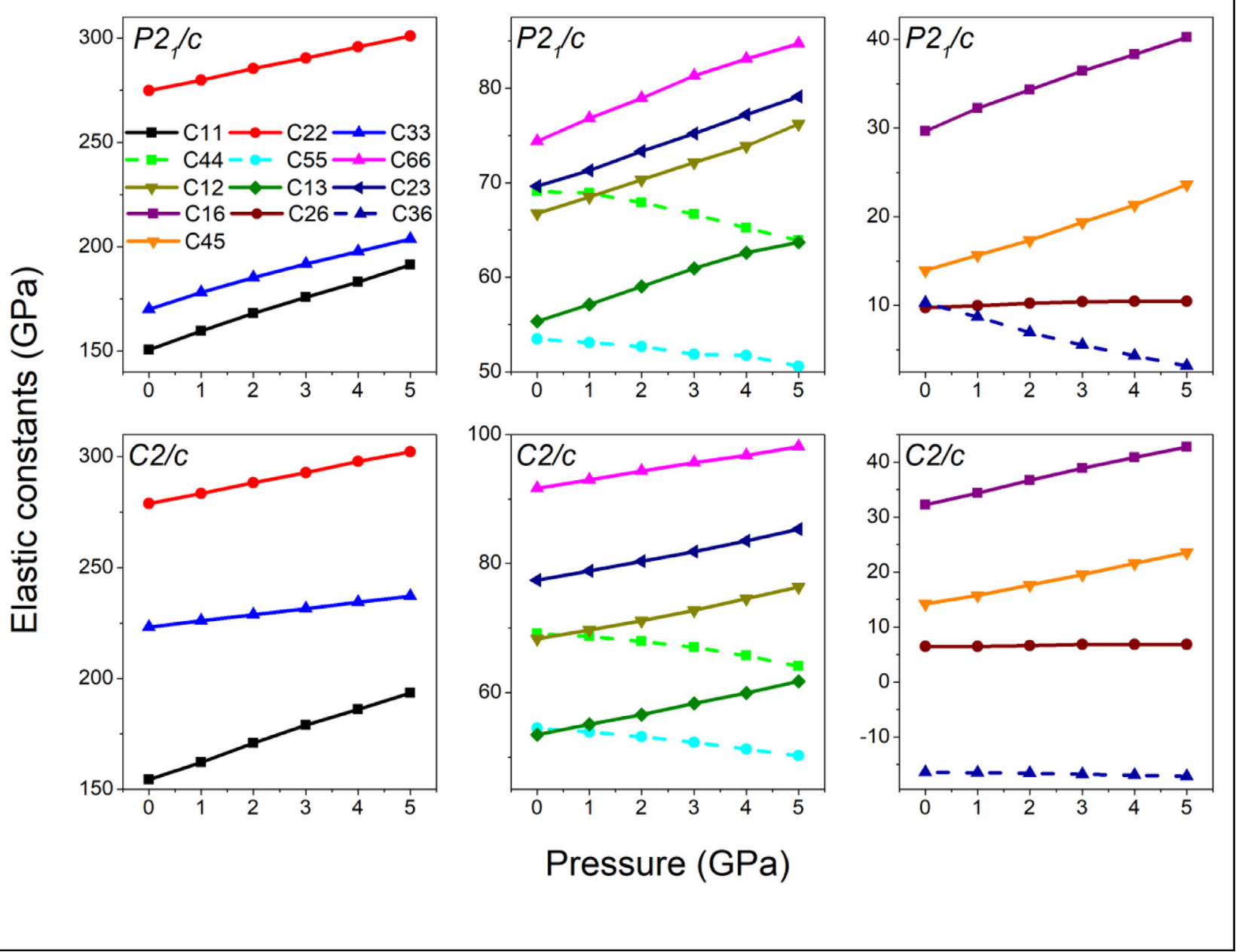

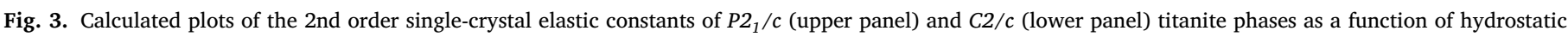

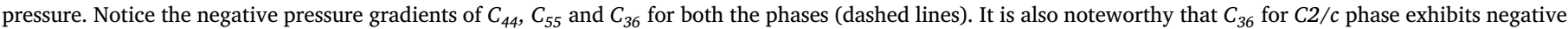
values and the negativity increases with increasing pressure.

drostatic pressures up to $5 \mathrm{GPa}$. The elastic constant tensor $\left(C_{i j}\right)$ consists of 13 independent components: $C_{11}, C_{22}, C_{33}, C_{44}, C_{55}, C_{66}, C_{12}, C_{12}$, $C_{23}, C_{16}, C_{26}, C_{36}$ and $C_{45}$. Fig. 3 presents their calculated values as a function of $p$, which, to the best of our knowledge, is completely a new theoretical data set. Unfortunately, no experimental data are available to validate our findings. The $P 2_{1} / c$ phase shows increasing magnitudes of the three compressional elastic constants: $C_{11}, C_{22}$ and $C_{33}$ with $p$, where $C_{11}$ and $C_{33}$ are nearly equal in the entire range of pressure considered for our calculations, but $C_{22}$ is always significantly higher than both $C_{11}$ and $C_{33}$. These compressional elastic constants are sensitive to $p$, shown by their large pressure derivatives $\left(C_{11}^{\prime}=8.16, C_{22}^{\prime}=5.21\right.$ and $\left.C_{33}^{\prime}=6.75\right)$. The other elastic constants: $C_{66}, C_{12}, C_{13}, C_{23}, C_{16}, C_{26}$ and $C_{45}^{33}$ hold positive relations with $p$ for both the phases. Among them, the elastic constants: $C_{66}$ and $C_{16}$ have the steepest gradients, where the pressure derivative of $C_{16}\left(C_{16}^{\prime}=2.11\right)$ is slightly higher than that of $C_{66}$ $\left(C_{66}^{\prime}=2.06\right)$. The other four constants: $C_{12}, C_{13}, C_{23}$ and $C_{45}$ have lower pressure derivatives $\left(C_{12}^{\prime}=1.89, C_{13}^{\prime}=1.67, C_{23}^{\prime}=1.89\right.$ and $\left.C_{45}^{\prime}=1.94\right)$. $C_{26}$ is almost unaffected by pressure $\left(C_{26}^{\prime}=0.15\right) . C_{13}$ is the most soft elastic constant of $P 2_{1} / c$ titanite phase, implying that the crystal would exhibit least resistance to the deformation along the $a$ direction under a longitudinal strain applied in the $c$ direction.

The $C 2 / c$ phase shows similar pressure dependent variations of the compressional elastic constants with $p\left(C_{11}^{\prime}=7.79, C_{22}^{\prime}=4.69\right.$ and $C_{33}^{\prime}=$ 2.78). $C_{33}$ shows less pressure dependent variations in $C 2 / c$ than $P 2_{1} / c$ titanite phase as the $c$ axis is less compressible in $C 2 / c$ (Table 1 ). Among the other elastic constants: $C_{66}, C_{12}, C_{13}, C_{23}, C_{16}, C_{26}$ and $C_{45}, C_{16}$ appears to be the most pressure sensitive constant $\left(C_{16}^{\prime}=2.11\right.$, whereas $C_{45}^{\prime}=1.87, C_{13}^{\prime}=1.67, C_{12}^{\prime}=1.59, C_{23}^{\prime}=1.58$ and $C_{66}^{\prime}=1.29$ ). In contrast, $C_{26}$ is virtually pressure independent elastic constant $\left(C_{26}^{\prime}=0.08\right)$, as in the $P 2_{1} / c$ phase. The elastic constant tensor shows a large difference between $C_{16}(32.2 \mathrm{GPa})$ and $C_{26}(6.47 \mathrm{GPa})$, suggesting that the crystal would have contrasting stiffness in the $a$ and $b$ directions under a strain applied on the $a b$ plane. It means that the titanite phase has the highest degree of anisotropy preferentially on the (001) plane.

Our calculations lead to a novel finding on the elastic behaviour of titanite. Among its 13 elastic constants, the three elastic constants: $C_{44}$, $C_{55}$ and $C_{36}$ show remarkably negative pressure gradients for both $P 2_{1} / c$ as well as $C 2 / c$. The crystals, thus, can undergo shear softening at elevated hydrostatic pressures, implying that they undergo shear softening at elevated pressures and become mechanically unstable at higher $p$. In case of $P 2_{1} / c$ phase, we obtain the steepest negative gradients for $C_{36}$ $\left(C_{36}^{\prime}=-1.43\right)$, whereas the lowest for $C_{55}\left(C_{55}^{\prime}=-0.56\right) . C_{44}$ also has high negative pressure derivatives $\left(C_{44}^{\prime}=-1.03\right)$. The pressure derivatives of these constants for $C 2 / c$ phase are: $C_{44}^{\prime}=-0.99, C_{55}^{\prime}=-0.85$ and $C_{36}^{\prime}=-$ 0.14 . It is noteworthy that $C_{36}$ is a negative elastic constant $(-16.41 \mathrm{GPa}$ at static condition), and its magnitude decreases with increasing $p$.

We now focus upon the physical implications of pressure induced shear softening behaviour of titanite phases. The pressure dependent acoustic mode softening is consistent with softening of $C_{44}$ as its softening has indirect influence on the structural instability [21]. On the other 


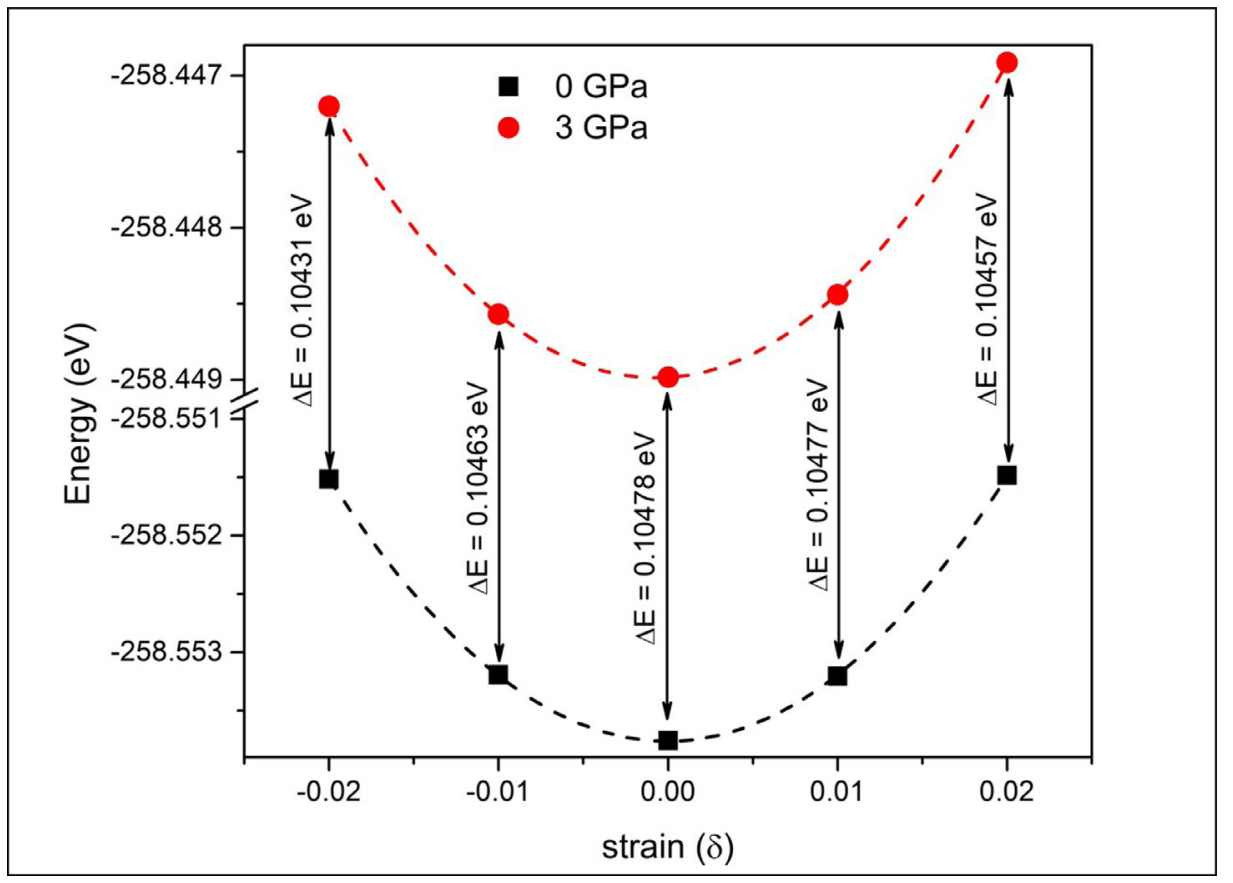

Fig. 4. Variations of energy with strains ( $\pm 2 \%)$ applied to the unstrained titanite crystal $\left(P 2_{1} / c\right.$ titanite) at $p=0$ (black) and 3 (red) GPa. This particular strain $\left[e=\left\{\delta^{2} /\left(1-\delta^{2}\right) ; 0 ; 0 ; 2 \delta ; 0 ; 0\right\}\right]$ was used to calculate $C_{44}$. The solid symbols denote the calculated values and the dotted lines represent 2 nd order best-fit. $p$ :hydrostatic pressure. hand, an elastic constant of a crystal can be positive if the energy in its strained state is higher than in its relaxed state. The elastic constants can be calculated from the energy of a strained crystal by expressed in a Taylor series expansion,

$E(V, \delta)=E\left(V_{0}, 0\right)+V_{0}\left(\sum_{i=1}^{6} \tau_{i} \delta_{i}+\frac{1}{2} \sum_{i=1}^{6} \sum_{j=1}^{6} C_{i j} \delta_{i} \delta_{j}+\mathrm{O}\left(\delta^{3}\right)\right)$

where $\delta$ is input strain $E(V, \delta)$ and $E\left(V_{0}, 0\right)$ are the energies corresponding to the strained and unstrained state of the crystal, respectively. Assuming the initial stress, $\tau_{i}=0$ in the unstrained crystal, we can extrapolate the following relation from Eq. (1),

$C_{i j} \propto \frac{\Delta E}{V_{0}}$

$\Delta \mathrm{E}$ is the energy difference between the strained and unstrained states, given by

$\Delta E=E(V, \delta)-E\left(V_{0}, 0\right)$

Now, let's consider $\left(C_{i j}\right)_{p_{1}}$ and $\left(C_{i j}\right)_{p_{2}}$ are the two values of a given elastic constant at pressure $p_{1}$ and $p_{2}$, respectively, where $p_{1}<p_{2}$. Using Eq. (2) we can write

$\frac{\left(C_{i j}\right)_{p_{1}}}{\left(C_{i j}\right)_{p_{2}}}=\frac{\Delta E_{p_{1}}}{\Delta E_{p_{2}}} \frac{\left(V_{0}\right)_{p_{2}}}{\left(V_{0}\right)_{p_{1}}}$

As $\left(V_{0}\right)_{p_{1}}>\left(V_{0}\right)_{p_{2}}$ for $p_{1}<p_{2}$, the negative pressure gradient of $C_{i j}$, i.e., $\left(C_{i j}\right)_{p_{1}}>\left(C_{i j}\right)_{p_{2}}$ demands, $\Delta E_{p_{1}}>\Delta E_{p_{2}}$. From Eq. (3),

$E_{p_{1}}\left(V_{p_{1}}, \delta\right)-E\left(\left(V_{0}\right)_{p 1}, 0\right)>E_{p_{2}}\left(V_{p_{2}}, \delta\right)-E\left(\left(V_{0}\right)_{p_{2}}, 0\right)$

which simplifies to

$E\left(\left(V_{0}\right)_{p_{2}}, 0\right)-E\left(\left(V_{0}\right)_{p_{1}}, 0\right)>E_{p_{2}}\left(V_{p_{2}}, \delta\right)-E_{p_{1}}\left(V_{p_{1}}, \delta\right)$

$\Rightarrow \Delta E\left(V_{0}, 0\right)_{p_{2}-p_{1}}>\Delta E_{\delta}\left(V_{\delta}, \delta\right)_{p_{2}-p_{1}}$

Eq. (5) indicates that the energy differences $\left(\Delta E\left(V_{0}, 0\right)_{p_{2}-p_{1}}\right)$ due to the pressure difference $\left(p_{2}-p_{1}\right)$ for the unstrained crystals must be higher than the strain $(\delta)$ induced energy difference $\left(\Delta E_{\delta}\left(V_{\delta}, \delta\right)_{p_{2}-p_{1}}\right)$. This is the necessary and sufficient condition for an elastic constant to attain a negative pressure gradient. Fig. 4 shows strain-energy curves which are used to calculate $C_{44}$ at 0 and $3 \mathrm{GPa}$ for the $P 2_{1} / c$ phase.
The energy difference, $\Delta E\left(V_{0}, 0\right)_{p_{2}-p_{1}}$ for the unstrained titanite crystal is $0.10478 \mathrm{eV}$, which is higher than any $\Delta E_{\delta}\left(V_{\delta}, \delta\right)_{p_{2}-p_{1}}$ of strained crystal for any nonzero value of $\delta$.

\subsection{The negative elastic constant}

An elastic constant can be negative, only when $\Delta E$ (Eq. (3)) becomes negative. We can say that a strained state of titanite crystal has lower energy than its relaxed state, which indicates the system is losing some energy by acquiring strain, tending to attain a different energy minimum. It suggests that the ground state structure is already internally strained (distorted), as observed by Marcherek and Fischer [32]. On the other hand, an elastic constant denotes a ratio between the resistive stresses developed in a body and the applied strain. The mechanical action can yield a negative value of the elastic constant when a compressive stress (negative quantity) is produced under extensional strain (positive quantity) or vice-versa. However, this is not a usual mechanical behaviour of solids. Consider an alternative approach to demonstrate the physical implication of negative elasticity. An isotropic body (which shows positive elasticity) under a uniaxial compression undergoes an expansion in directions perpendicular to the compression. In other words, the body compressed in a particular direction develops contractional strain (negative) in the applied compression direction, and extensional strain (positive) across it. But, a crystal with negative elasticity can develop contractional strain both along and across the compression direction. We face a similar scenario in titanite. The application of positive strains yields negative stresses in $C 2 / c$ titanite. Such negative elasticity was first experimentally reported by Boppart et al. [28] in 1980 from sound velocity measurements, and later by Schärer and Wachter [30] from the Brillouin scattering of $\mathrm{Sm}_{\mathrm{x}} \mathrm{La}_{1-\mathrm{x}} \mathrm{S}$. Both these studies obtained negative values of $C_{12}$ in cubic phases at room temperature. However, they did not provide any specific atomic scale mechanism to explain this uncommon elastic behaviour. In fact, their results indicate the crystal symmetry is not a key factor to the negative elasticity. In this study we take into account the bond kinematics during crystal deformation to address this unique mechanical problem.

$C_{36}$ was calculated by applying shear strain $\varepsilon_{12}$, in the monoclinic structure of titanite. The stress components along $a, b$ and $c$ directions were obtained as a function of input shear strain to find $C_{36}$. We take the 


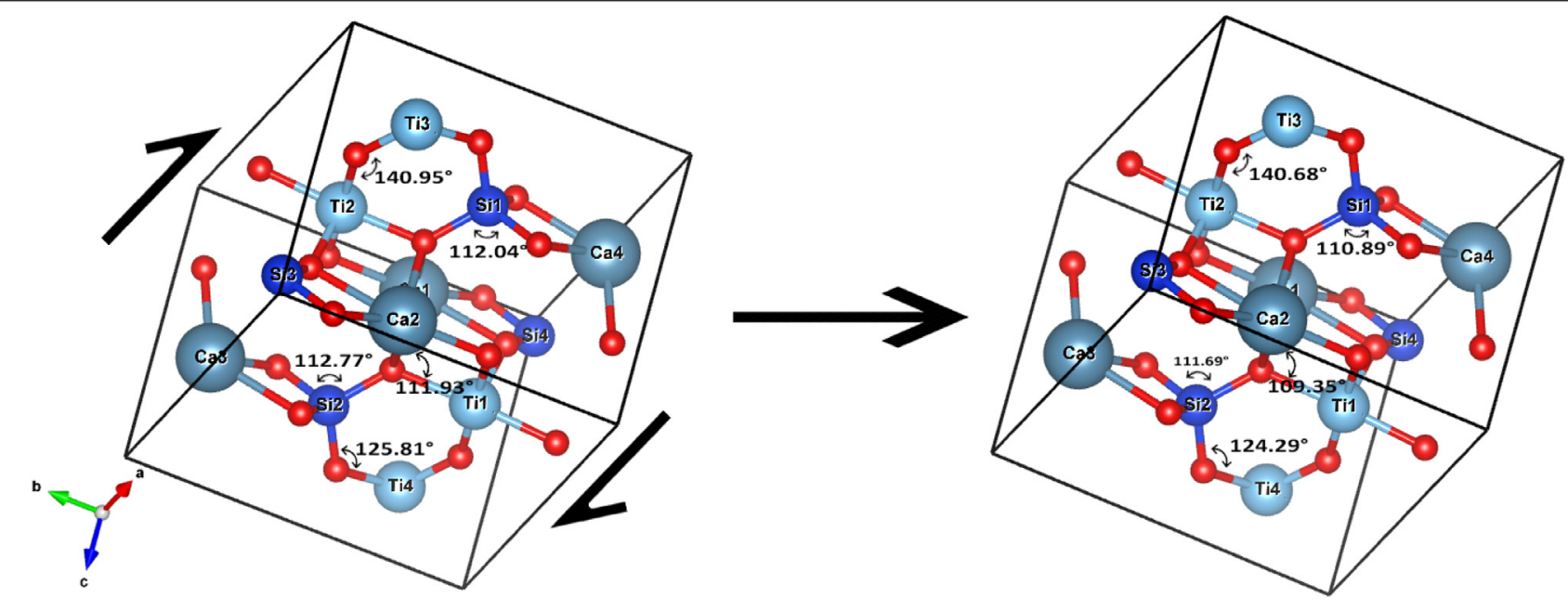

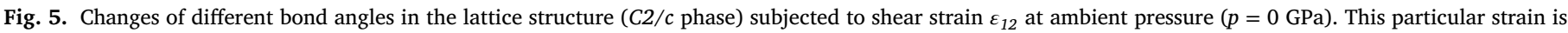
associated with $C_{36}$.

stress component $\sigma_{33}$ corresponding to the applied shear strain, $\varepsilon_{12}$. According to our study, the shear strain $\varepsilon_{12}$ develops compressive stresses: $\sigma_{11}, \sigma_{22}$ and $\sigma_{33}$ along the three crystallographic directions [100], [010] and [001], respectively. It is noteworthy that the crystal gives rise to the negative elastic constants essentially under shear deformation. The role of shear strain in lattice scale modifications thus appears to be a key in theorizing the mechanical behaviour of titanite. We propose a bond dynamic model to demonstrate how the lattice collapse by bond rotation can lead to such negative elasticity. This model allows us to suggest the bond kinematics as a driving factor for structural contraction perpendicular to the compression direction. The rotational kinematics is evidently manifested in the relative structural rearrangement of the constituent neighbouring polyhedra, which can be quantified by the angular relations between two neighbouring polyhedra. The structure can accommodate the compressive stress entirely by the rotational motion of rigid bonds. Evidently, this rotational kinematics involves complex dynamics of the rigid bonds, which are supposed to be tangled in a network. They can intricately influence one another in their rotational motion. This mode of rotation-dominated bond kinematics seems to restrict structural expansion perpendicular to the direction of applied compression, as in the case of positive elasticity. In contrary, it results in contraction perpendicular to the compression direction, satisfying the condition required for negative elasticity. It follows from this discussion that the rotational bond kinematics is the key factor in controlling the negative elasticity in titanite crystals.

To support the theoretical interpretation, we performed an analysis of bond angles in the unstrained and strained states of the lattice configuration of the actual crystal structure of $C 2 / c$ titanite (Fig. 5). For a given Lagrangian strain $( \pm 2 \%)$, the $\mathrm{Ca}-\mathrm{O}-\mathrm{Si}$ bond angle is found to change from its initial value of $95.18^{\circ}$ to $97.35^{\circ}$, implying a bond rotation by $2.17^{\circ}$. Similarly, Ca-O-Ti and Ti-O-Si bond angles are reduced from $111.93^{\circ}$ to $109.35^{\circ}$ and $125.81^{\circ}$ to $124.29^{\circ}$, respectively. The two bonds thus undergo substantial rotations, $2.58^{\circ}$ and $1.52^{\circ}$, respectively. These observations confirm that the given shear strain is accommodated dominantly by angular reorientation of cationic polyhedral bonds. In case of the elastic constant, $C_{36}$, the applied shear strain (on $a b$ plane in the $a$-direction) affects the $\mathrm{O}-\mathrm{O}$ distance to a small extent, as measured on that shear plane. For a variation of the shear strain between -0.02 and 0.02 , the $\mathrm{O}-\mathrm{O}$ distance undergoes negligibly small changes $(2.737$ $\AA$ to $2.723 \AA$ ). On contrary, the $\mathrm{O}-\mathrm{Si}-\mathrm{O}$ angles in $\mathrm{SiO}_{4}$-tetrahedra, measured on planes subparallel to the $a b$-plane are reduce from $112.035^{\circ}$ to $110.891^{\circ}$. The neighbouring Ti-polyhedra also undergo an angular rearrangement, involving the bridging bond angle of $\mathrm{Ti}-\mathrm{O}-\mathrm{Ti}$ between the tetrahedra to vary from $140.958^{\circ}$ to $140.682^{\circ}$. Our analysis clearly reveals that, in contrary to the natural behaviour of crystals to resist the strain, the deformation appears to be faciliated by the crystal itself. This antipodal mechanical behaviour of the crystal is a reflection of the negative elastic constants. Our analysis of the strain-induced bond angle modifications in titanite strongly supports the bond rotation as a mechanism to determine the negative elasticity of a crystal. We envisage bond stretching and bond rotation as two competing mechanisms in determining the positive versus negative elasticity of crystalline solids. The dominance of bond stretching over bond rotation would result in a positive elastic behaviour, as commonly described in solid mechanics. However, the opposite bond dynamics, i.e., restricted bond stretching will favour the negative elasticity, as in the case of titanite phase. It is noteworthy that the negative elastic constant $\left(C_{36}\right)$ of $C 2 / c$ titanite increases its magnitudes with increasing pressure (Fig. 3). This pressure-induced enhancement of the negative behaviour warrants our interpretation. Increasing pressures generally strengthen atomic bonds in a crystal and restrict their stretching during deformation under a deviatoric stress field. At elevated pressures the crystal thus prefers to accommodate the strain mainly by bond rotation and facilitate its negative elastic behaviour.

To explain the rotational bond dynamics responsible for the negative $C_{36}$, we have calculated the valence charge density on the three principal crystallographic planes: (100), (010), and (001) at both ambient and high pressures (Fig. 6). The charge distribution clearly reveals the anisotropic nature of titanite. In the (001) plane, $\mathrm{Ti}-\mathrm{O}$ shows strong covalent bonding with a maximum accumulated charge of 8.4148 $\mathrm{e} / \AA^{3}$ at $0 \mathrm{GPa}$, which is the highest among all other planes. The magnitude of charge accumulation on this plane increases further to 8.521 $\mathrm{e} / \AA^{3}$ at $5 \mathrm{GPa}$. In contrast, it decreases with pressure on (100). Such a large accumulation of the electronic charge on (001) gives rise to high bond strength between $\mathrm{Ti}$ and $\mathrm{O}$ atoms, which favours the bond rotation kinematics to dominate over bond stretching/ shortening under a given shear strain $\varepsilon_{12}$. The shear strain is eventually accommodated by local rotation of the atom clusters (Ti-O) present in this plane, resulting in a contraction of the whole structure, a condition for $C_{36}$ to attain a negative value. Furthermore, the strong Ti-O covalent bond produces large resistance to applied strain $\varepsilon_{22}$ in the crystal. The charge density plot then allows us to explain why $C_{22}$ turns out to be the numerically largest component of the elastic constant tensor $C_{i j}$ of titanite (Fig. 3). 


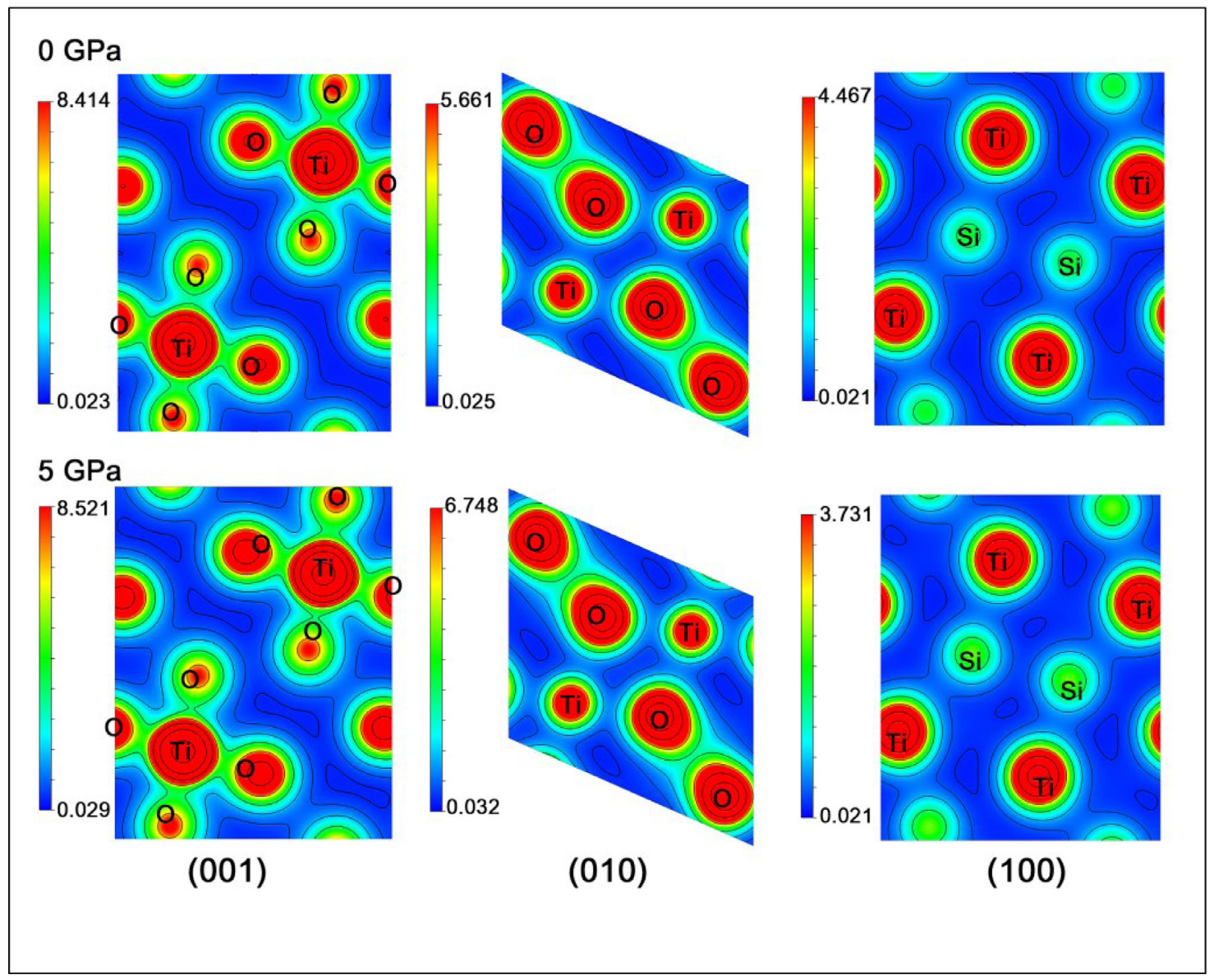

Fig. 6. Valence charge density of the $C 2 / c$ titanite phase on the three crystallographic planes: (001), (010) and (100) at ambient and high pressure. It is noteworthy that the charge density on (001) displays strong covalent bonding between Ti and O. Moreover, this crystallographic plane shows a much higher degree of anisotropy in charge distribution than the other two planes. The charge accumulation is reduced on the (001) plane at high pressure.

\subsection{Elastic moduli}

We also tested the mechanical stability of the monoclinic titanite phase using the following stability criteria, described by Börn [47],

$$
\begin{gathered}
C_{11}>0, C_{22}>0, C_{33}>0, C_{44}>0, C_{55}>0, C_{66}>0, \\
{\left[C_{11}+C_{22}+C_{33}+2\left(C_{12}+C_{13}+C_{23}\right)\right]>0} \\
\left(C_{33} C_{66}-C_{36}^{2}\right)>0,\left(C_{44} C_{55}-C_{45}^{2}\right)>0,\left(C_{22}+C_{33}-2 C_{23}\right)>0
\end{gathered}
$$

Our analysis reveals that the negative elastic constant does not affect the mechanical stability of the phase, even at high pressures.

According to the standard equations used in the theory of elasticity, the elastic constants $\left(C_{i j}\right)$ are the fundamental quantities to determine the elastic moduli of a crystal. The negative values of the elastic constant can thus influence the bulk mechanical properties. We evaluated the bulk $(B)$, shear $(G)$ and Young's $(E)$ moduli of both titanite phases as a function of pressure, accounting both positive and negative elastic constants (Fig. 7). Our calculations yield very low shear modulus for both the titanite phases, which is consistent with the results $(G \sim 46-52$ $\mathrm{GPa}$ ) of Salje et al. [14]. However, the calculated $G$ values ( $\sim 63 \mathrm{GPa})$ at static condition $(T=0)$ is slightly higher. We found the value of $B(102$ $\mathrm{GPa}$ at static condition) for the $P 2_{1} / c$ phase, which lies in the range of values obtained from experiments with damaged (85 GPa) [14] and undamaged (131 GPa) [15] titanite. $B$ is the most pressure sensitive elastic modulus, where $B^{\prime}=3.63$ in the pressure range 0 to $5 \mathrm{GPa}$. In contrast, $E$ and $G$ are much less sensitive to pressure $\left(E^{\prime}=1.9\right.$ and $\left.G^{\prime}=0.52\right) . C 2 / c$ phase shows similar pressure dependent behaviour of $B$ and $E\left(B^{\prime}=2.98\right.$, $\left.E^{\prime}=0.59\right)$, whereas $G$ is less sensitive to pressure $\left(G^{\prime}=0.06\right)$. The static shear and bulk modulus are slightly higher than those of $P 2_{1} / c$ phase 69 and $112 \mathrm{GPa}$, respectively). The high values of bulk modulus indicate the

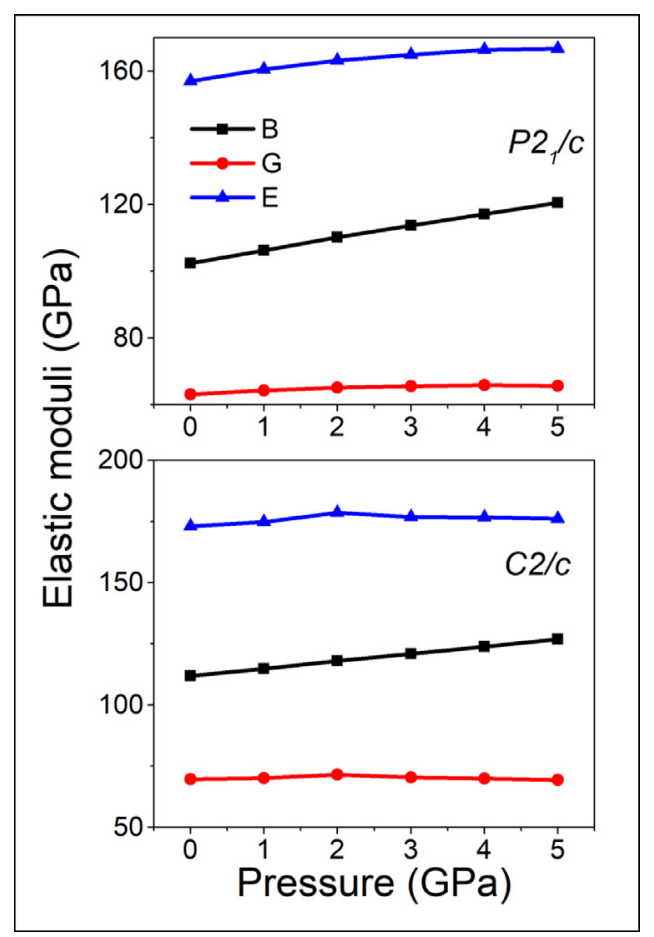

Fig. 7. Variation of the elastic moduli: bulk $(B)$, shear $(G)$ and Young's $(E)$ moduli of the titanite phases with hydrostatic pressures ( 0 to $5 \mathrm{GPa}$ ). $B$ is found to be the most sensitive modulus to pressure. 


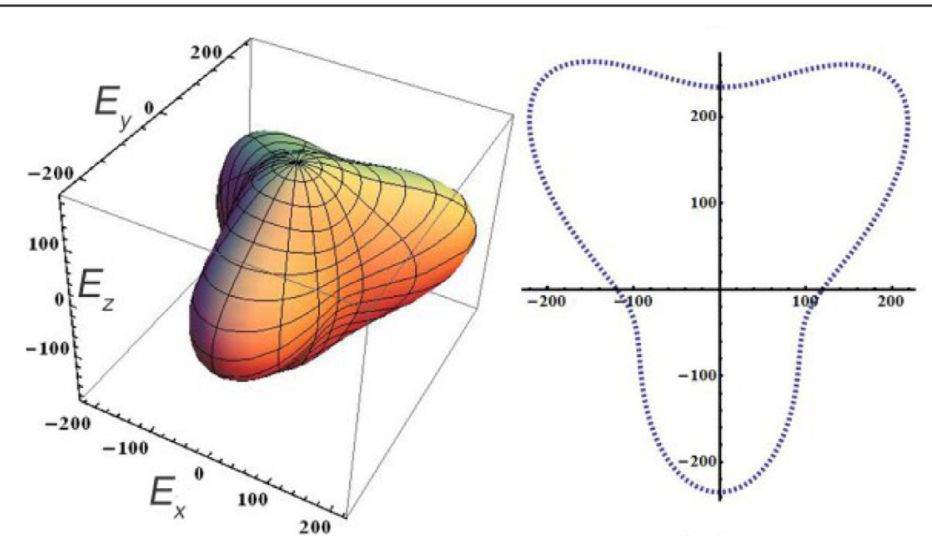

(001)
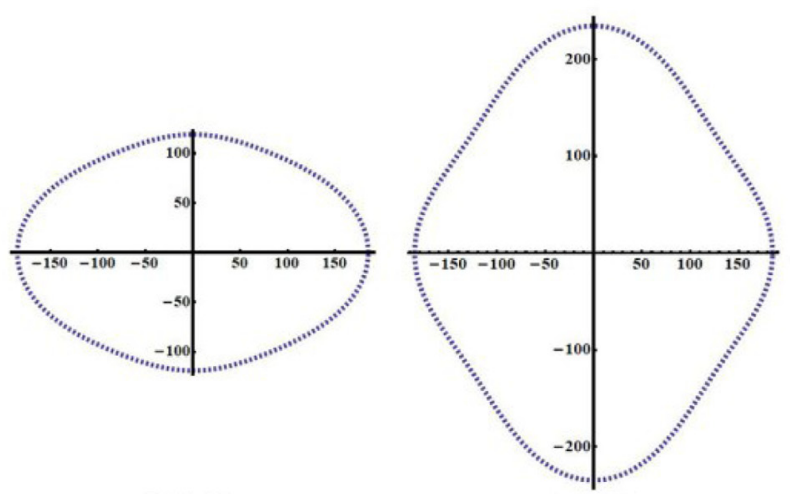

(010)

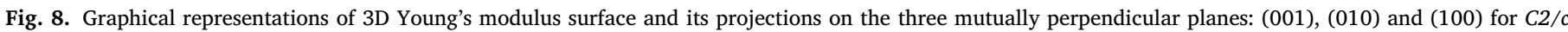
titanite. The anisotropy is clearly visible on (001) and it conforms to the valence charge density (See Fig. 6).

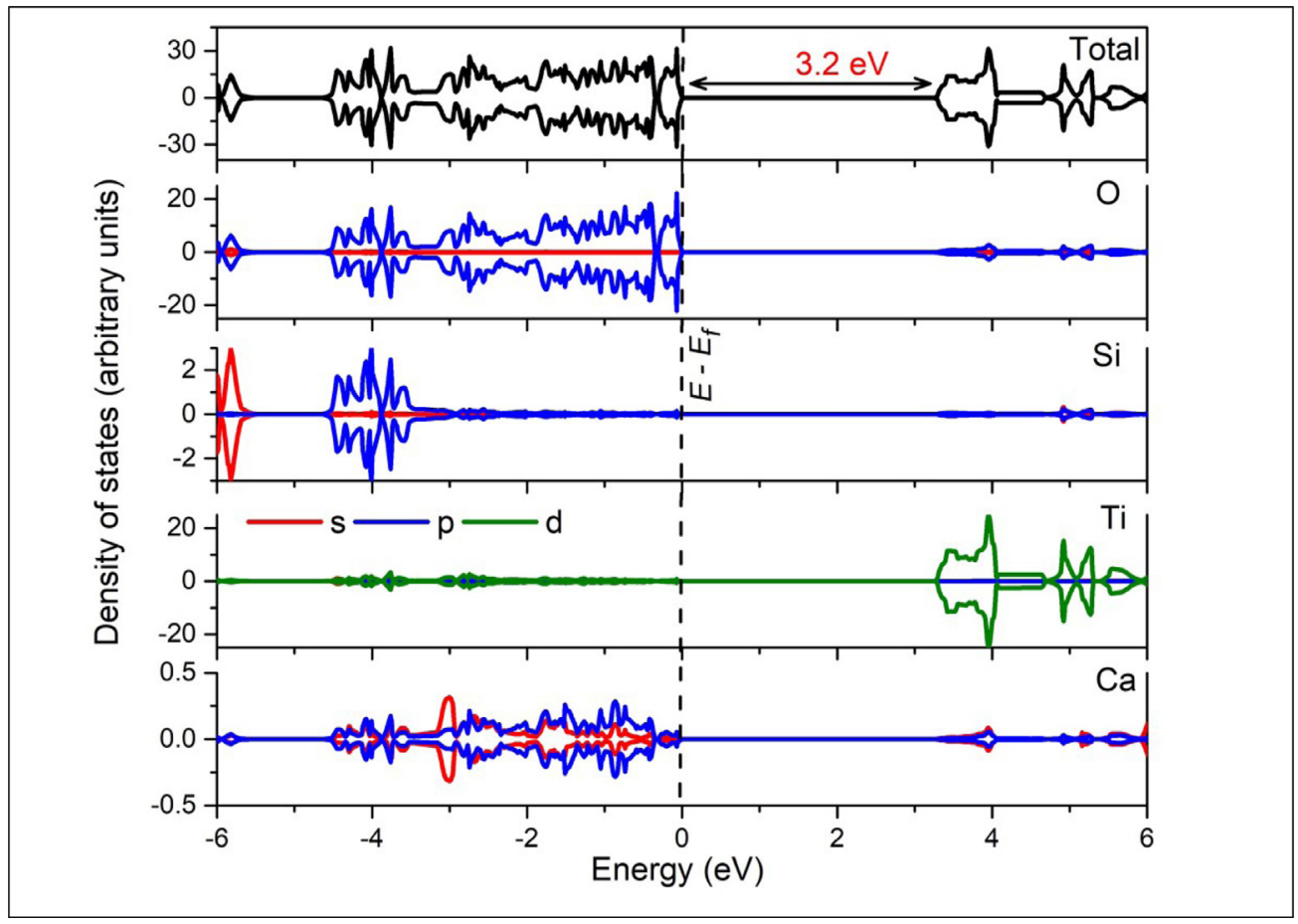

Fig. 9. Spin polarised orbital projected electronic density of states for $P 2_{1} / c$ titanite phase.

titanite phase can be used as a hard material, despite its directional negative single crystal elasticity. Our theoretical results also predict titanite as an example of perfectly anisotropic crystal with universal anisotropy index [48] of 0.69. A 3D Young's modulus surface, which is also a measure of minimum thermal conductivity, is constructed for $C 2 / c$ titanite to show the degree of its elastic anisotropy (Fig. 8). This 3D surface of $E$ vividly reveals strongly anisotropic mechanical behaviour of titanite even under static condition, with $E_{1}=119.48, E_{2}=234.29$ and $E_{3}=$ 186.17 GPa, where $E_{1}, E_{2}$ and $E_{3}$ are the Young's modulus in the $a, b$ and $c$ direction, respectively. The average Young's modulus $(E=173.11)$ is very close to $E_{3}$, implying that the (001) plane plays the most crucial role in determining the bulk mechanical behaviour of titanite.

We consider the ratios of $E_{1}, E_{2}$ and $E_{3}$ to express the degree of anisotropy on the three principal crystallographic planes: (001), (010) and (100), where $E_{2} / E_{1}=1.96, E_{3} / E_{1}=1.56$, and $E_{2} / E_{3}=1.17$, respectively, (Fig. 8). The calculated ratios suggest the highest anisotropy on (001) and the lowest on (100), which is consistent with the valence charge density (Fig. 6). The charge distributions show much stronger directionality on (001) than the other two planes, resulting in the highest degree of anisotropy in Young's modulus on this plane.

\subsection{Electronic properties}

The electronic density of states determines both intraband and interband transition, which influence the optical properties of materials. The spin polarized electronic density of states for $P 2_{1} / c$ titanite is presented in Fig. 9. Fermi level is set to zero of the energy scale. The electronic energy gap $\left(E_{g}\right)$ is found to be $3.2 \mathrm{eV}$, which indicates titanite can be used as semiconductor. The valence band is characterized by $O-p, C a-s$ and $C a-p$ states. The top of the valence band, i.e., closer to Fermi energy, is mainly dominated by $O-p$ states. However, $T i-d$ states are the principal 


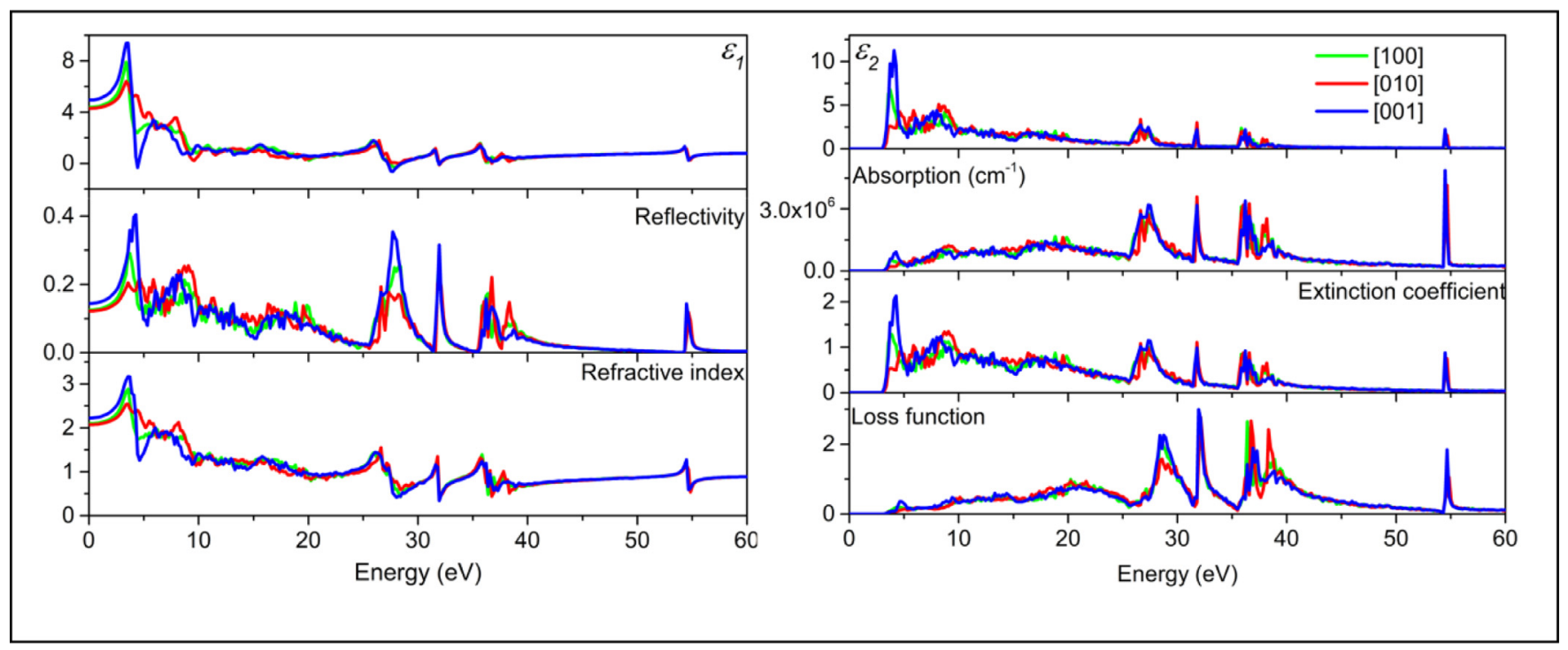

Fig. 10. Polarization dependent optical properties of $P 2_{1} / c$ titanite phase as a function of energy.

contributor of the bottom of the conduction band. Some hybridization between $O-p$ and $T i-d$ also can be observed in the conduction band.

\subsection{Optical properties}

Using the electronic structure we calculated frequency dependent real $\left(\varepsilon_{1}\right)$ and imaginary $\left(\varepsilon_{2}\right)$ part of the complex dielectric function of $P 2_{1} / c$ titanite (Fig. 10), which provide a new set of findings on the optical properties of this silicate phase. The dielectric function is resolved into three polarization direction [100], [010] and [001], i.e., parallel to $a, b$ and $c$-axis, respectively, as titanite belongs to highly anisotropic monoclinic crystal class. We obtain the static dielectric constant (i.e., at $\omega=0$ ) values as 4.38, 4.29 and 4.94, corresponding to $a$-, $b$ - and $c$ axis respectively. Their highest peak values, 8.04, 6.43 and 9.47 occur at $\sim 3.36 \mathrm{eV}$. The real part of the dielectric function is strongly anisotropic. $\varepsilon_{1}$ shows a sharp decrease as we move from $3.36 \mathrm{eV}$ to a higher frequency region and $\varepsilon_{1}$ parallel to $c$ attains a negative value at $\sim 4.36$ $\mathrm{eV}$, which indicates that most of the incident photon along $c$ will be reflected at this point. The result suggests that this metallic behaviour of titanite can be used for shielding purpose in a specific frequency region. We evaluated the electron loss function to study the plasmon resonance where their frequency peaks correspond to the metallic $\left(\varepsilon_{1}<0\right)$ to dielectric $\left(\varepsilon_{1}>0\right)$ transition. The loss function peaks occur exactly at a point where $\varepsilon_{1}$ switches from negative to positive $(\sim 4.36 \mathrm{eV})$. Several peaks are obtained in the range between 26 to $40 \mathrm{eV}$ and these high frequency peaks depict an excellent dielectric property of titanite. A decent knowledge of the refractive index of a material is pre-requisite to judge its suitability for potential optical applications. Here our calculation yield the refractive index $\left(\mathrm{n}_{0}\right)$ of titanite as $2.09,2.07$ and 2.21 for $a, b$ and $c$, respectively, fairly in agreement with previously reported values [40].

The complex part $\varepsilon_{2}$ represents the photon absorption. The optical absorption edge is found at $\sim 3.2 \mathrm{eV}$ for all the directions, suggesting the threshold for direct optical transition from valence band to conduction band at the Gamma point, which is consistent with the electronic band gap (Fig. 9).The highest peak on $\varepsilon_{2}$ that occurs at $4.8 \mathrm{eV}$ has a value of 11.3 , which justifies the reddish brown color of titanite. This peak corresponds to the hybridization between Ti-d and $O-p$ states (Fig. 9). Several peaks in the high frequency regions correspond to intra-band transition depending upon the energy of incident photon. Our calculated absorption spectra has a spread from $\sim 3.2 \mathrm{eV}$ to $60 \mathrm{eV}$. The same spread is also visible in reflectivity spectra with a maximum of 0.4 at $4.26 \mathrm{eV}$. For

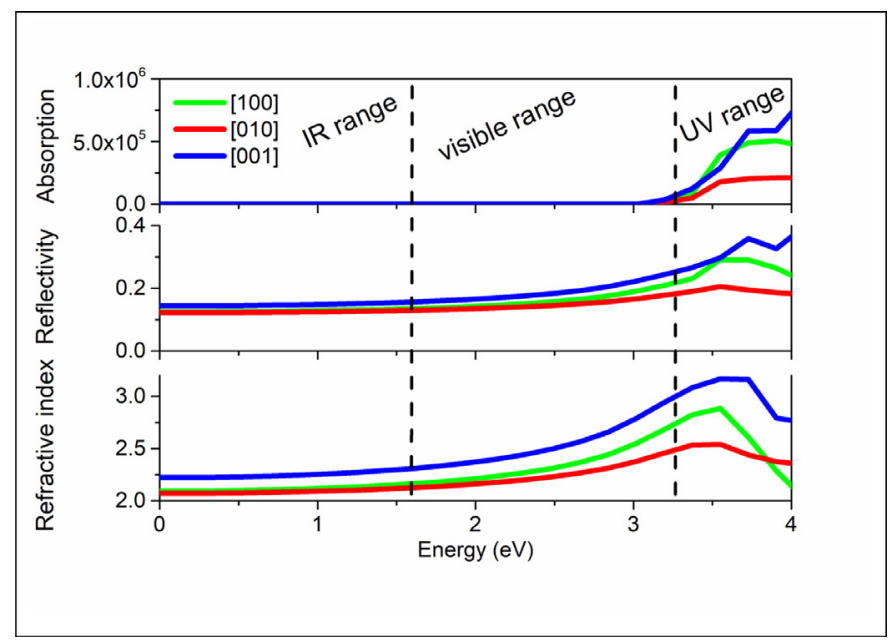

Fig. 11. Anisotropic absorption, reflectivity and refractive index of $P 2_{1} / c$ titanite in infrared (IR), visible and ultraviolet (UV) spectra.

Table 2

Static dielectric constants $\left(\varepsilon_{1}\right)$ and static refractive index $\left(\mathrm{n}_{0}\right)$ in principal crystallographic directions and anisotropy rate $\left(\mathrm{A}_{\mathrm{OPT}}\right)$ for $\mathrm{P} 2_{1} / \mathrm{c} \mathrm{ti}$ tanite.

\begin{tabular}{llll}
\hline Direction & $\varepsilon_{1}$ & $\mathrm{n}_{0}$ & $\mathrm{~A}_{\mathrm{OPT}}$ \\
\hline $\mathrm{x}$ & 4.381 & 2.094 & {$[0.966,0.983]$} \\
$\mathrm{y}$ & 4.291 & 2.072 & {$[0.946,0.973]$} \\
$\mathrm{z}$ & 4.937 & 2.223 & {$[1.088,1.043]$} \\
\hline
\end{tabular}

a comprehensive understanding, we have plotted absorption, reflectivity and refractive index spectra, covering the IR, visible and UV ranges (Fig. 11). The absorption and reflectivity increase rapidly as incident photon frequency crosses the visible spectrum range, especially in the [001] direction.

The optical anisotropy of titanite is quantified using the parameter ( $A_{O P T}$ ) given by Cherrad et al. [49]. The departure of $A_{O P T}$ from 1 indicates the degree of anisotropy. Table 2 presents $A_{O P T}$ values and their corresponding directional static dielectric constants $\left(\varepsilon_{1}\right)$ and refractive indices $\left(\mathrm{n}_{0}\right)$. These anisotropic optical properties are consistent with our 
previous findings. Extinction coefficient, which is a measure of the capability of light absorption of a material, also shows similar behaviour. Our calculated extinction coefficient shows a major peak at $4.26 \mathrm{eV}$ along with several minor peaks spread in the spectrum $0-60 \mathrm{eV}$. The results indicate that titanite behaves like an opaque material in the UV region due to its high absorption and reflectivity, whereas it is transparent in the visible range (up-to $\sim 3.2 \mathrm{eV}$ ).

\section{Conclusions}

To conclude the principal outcomes of this study, we have presented a completely new theoretical data set on the mechanical properties of monoclinic titanite phase. Our theoretical calculations reveal unusual negative values of the elastic constant: $C_{36}$ for $C 2 / c$ phase and negative pressure gradients of the shear elastic constants, $C_{44}, C_{55}$ and $C_{36}$ for both the titanite phase: $P 2_{1} / c$ and $C 2 / c$. A novel atomic scale mechanism is proposed to demonstrate the cause of negative elasticity in titanite. The rotational bond kinematics, driven by valence charge accumulation on (001) plane leads to structural collapse in a direction orthogonal to the applied shear strain, resulting in the negative elastic behaviour. Based on strain-energy calculations at varying pressures, we constrain the necessary conditions leading to the negative pressure gradients of the shear elastic constants. Our lattice dynamical analysis confirms the structural phase transition from $P 2_{1} / c$ to $C 2 / c$ in titanite within $5 \mathrm{GPa}$. We believe our theory will stimulate workers from different disciplines, especially the materials scientists to synthesize and characterize materials with exceptional mechanical properties.

Our findings suggest that wide bandgap $\left(E_{g}=3.2 \mathrm{eV}\right)$ titanite shows an exceptionally strong opacity in the UV region. We thus propose this silicate as a potential shield material for UV radiation. This crystalline phase can also be used for developing optical filters/polarizers. Its transparent property in the low-frequency region provides an excellent scope for designing various optoelectronic devices.

\section{Declaration of Competing Interest}

The authors declare that they have no known competing financial interests or personal relationships that could have appeared to influence the work reported in this paper.

\section{Acknowledgments}

We thank two anonymous reviewers and Editor Prof. Z. Mao for their constructive comments and suggestions for improving the scientific content of this work. We grateful to Dr. C.E Mohn, UiO for his help on the language correction. PKD thanks the Centre for Earth Evolution and Dynamics (CEED), funded by CoE-grant 223272 from the Research Council of Norway. SKM gratefully acknowledges the Department of Science and Technology, Govt. of India for awarding him a research fellowship. NM expresses his gratitude to the BRNS and SERB-DST, Govt. of India for providing him the research funding (Sanction No. 36(2)/14/25/2016BRNS) and the JC Bose fellowship (SR/S2/JCB-36/2012), respectively.

\section{References}

[1] W.A. Deer, R.A. Howie, J. Zussman, Rock forming minerals, volume 1A: orthosilicates, Geol. Soc. 1 (1982) 919.

[2] E.R. Vance, D.K. Agrawal, Incorporation of radionuclides in crystalline titanates, Nucl. Chem. Waste Manag 3 (1982) 229-234.

[3] P.J. Hayward, F.E. Doern, E.V. Cecchetto, S.. Mitchell, Leaching studies of natural and synthtic titanite, a potential host for wastes from the reprocessing of canadian nuclear fuel, Can. Minerol 21 (1983) 611-623.

[4] Y. Pan, M.E. Fleet, N. MacRae, Late alteration in titanite (CaTiSiO5): Redistribution and remobilization of rare earth elements and implications for $\mathrm{U} / \mathrm{Pb}$ and $\mathrm{Th} / \mathrm{Pb}$ geochronology and nuclear waste disposal, Geochim. Cosmochim. Acta. 57 (1993) 355-367.

[5] R. Rao, T. Sakuntala, P. Sengupta, G.K. Dey, K. Sharma, G.P. KothiyaI, High pressure behavior of sphene glass ceramics, Board Res. Nuclear Sci., India (2008).

[6] B.R. Frost, K.R. Chamberlain, J.C. Schumacher, Sphene (titanite): phase relations and role as a geochronometer, Chem. Geol 172 (2001) 131-148.
[7] S. Rath, Comparative pressure-dependent structural studies of titanite (CaTiOSiO4) and Malayaite (CaSnOSiO4), 2002.

[8] D.H. Lindsley, D. Hausermann, High-pressure phase transition in titanite (CaTiOSi04), 81 (1996) 1527-1530.

[9] C.L. Hollabaugh, W.G. College, The crystal structure of an AI-rich titanite from Grisons, Switzerland, 69 (1984) 725-732.

[10] R.J. Angel, M. Kunz, R. Miletich, A.B. Woodland, M. Koch, D. Xirouchakis, Highpressure phase transition in CaTiOSiO4 titanite, Phase Transitions 68 (1999) 533543, doi:10.1080/01411599908224532.

[11] M. Zhang, E.K.H. Salje, U. Bismayer, H.G. Unruh, B. Wruck, C. Schmidt, Phase transition(s) in titanite CaTiSiO5: an infrared spectroscopic, dielectric response and heat capacity study, Phys. Chem. Miner. 22 (1995) 41-49, doi:10.1007/BF00202679.

[12] M. Taylor, G.E. Brown, High-temperature structural study of the P21/a e A2/a phase transition in synthetic titanite, CaTiSiO5, Am. Mineral. 61 (1976) 435-447.

[13] M.J. Gutmann, K. Refson, M.V. Zimmermann, I.P. Swainson, A. Dabkowski, H. Dabkowska, Room temperature single-crystal diffuse scattering and ab initio lattice dynamics in CaTiSiO5, J. Phys. Condens. Matter. (2013) 25, doi:10.1088/0953-8984/25/31/315402.

[14] E.K.H. Salje, D.J. Safarik, J.C. Lashley, L.A. Groat, U. Bismayer, Elastic softening of metamict titanite CaTiSiO5: Radiation damage and annealing, Am. Mineral. 96 (2011) 1254-1261, doi:10.2138/am.2011.3747.

[15] R.J. Angel, M. Kunz, R. Miletich, A.B. Woodland, M. Koch, R.L. Knoche, Effect of isovalent $\mathrm{Si}$, Ti substitution on the bulk moduli of $\mathrm{Ca}(\mathrm{Ti}(1-\mathrm{x}) \mathrm{Si}(\mathrm{x}))$ SiO5titanites, Am. Mineral. 84 (1999) 282-287, doi:10.2138/am-1999-0310.

[16] S. Fu, J. Yang, J.F. Lin, Abnormal elasticity of single-crystal magnesiosiderite across the spin transition in earth's lower mantle, Phys. Rev. Lett. 118 (2017) 16, doi:10.1103/PhysRevLett.118.036402.

[17] S. Speziale, T.S. Duffy, R.J. Angel, Single-crystal elasticity of fayalite to $12 \mathrm{GPa}$, J. Geophys. Res. B Solid Earth. 109 (2004) 1-15, doi:10.1029/2004JB003162.

[18] R.J. Angel, J.M. Jackson, H.J. Reichmann, S. Speziale, Elasticity measurements on minerals: a review, Eur. J. Mineral. 21 (2009) 525-550, doi:10.1127/0935-1221/2009/0021-1925.

[19] R.A. Casali, N.E. Christensen, Elastic constants and defromations potential of ZnS and ZnSe under pressure, Solid State Commun 108 (1998) 793-798.

[20] B.B. Karki, M.C. Warren, L. Stixrude, G.J. Ackland, J. Crain, Ab initio studies of highpressure structural transformations in silica, Phys. Rev. B. 55 (1997) 3465-3471, doi:10.1103/PhysRevB.55.3465.

[21] P.K. Das, N. Mandal, A. Arya, Effects of Fe substitution on B3-B1 phase transition and structural, vibrational, and electronic properties of ZnS from DFT calculations, J. Appl. Phys. 121 (2017) 085101, doi:10.1063/1.4976813.

[22] P.K. Das, N. Mandal, A. Arya, Effects of cation ordering on the elastic and electronic properties of Mg-Fe silicate phases at high pressures, J. Appl. Phys. 122 (2017) 225107.

[23] P.K. Das, A. Chowdhury, N. Mandal, A. Arya, First-principles characterisation of the pressure-dependent elastic anisotropy of SnO 2 polymorphs, Philos. Mag. 96 (2016) 1861-1882, doi:10.1080/14786435.2016.1177228.

[24] G.W. Milton, A.V Cherkaev, Which Elasticity Tensors are Realizable? J. Eng. Mater. Technol. 117 (1995) 483-493.

[25] J.N. Grima, R. Gatt, A. Alderson, K.E. Evans, On the origin of auxetic behaviour in the silicate $\alpha$-cristobalite, J. Mater. Chem. 15 (2005) 4003-4005 https://doi.org/10.1039/B508098C.

[26] J.W. Jiang, H.S. Park, Negative poisson's ratio in single-layer black phosphorus, Nat. Commun. 5 (2014) 1-7, doi:10.1038/ncomms5727.

[27] L. Yu, Q. Yan, A. Ruzsinszky, Negative Poisson's ratio in 1T-type crystalline two-dimensional transition metal dichalcogenides, Nat. Commun. 8 (2017) 1-8, doi:10.1038/ncomms15224.

[28] H. Boppart, A. Treindl, P. Wachter, S. Roth, First observation of a negative elastic constant in intermediate valent TmSe, Solid State Commun 35 (1980) 483-486, doi:10.1016/0038-1098(80)90253-7.

[29] Y.C. Zhou, C. Zhao, F. Wang, Y.J. Sun, L.Y. Zheng, X.H. Wang, Theoretical prediction and experimental investigation on the thermal and mechanical properties of bulk $\beta$ Yb2Si2O7, J. Am. Ceram. Soc. 96 (2013) 3891-3900, doi:10.1111/jace.12618.

[30] U. Schärer, P. Wachter, Negative elastic constants in intermediate valent SmxLa1-xS, Solid State Commun 96 (1995) 497-501, doi:10.1016/0038-1098(95)00418-1.

[31] Z.J. Wu, E.J. Zhao, H.P. Xiang, X.F. Hao, X.J. Liu, J. Meng, Crystal structures and elastic properties of superhard Ir N2 and Ir N3 from first principles, Phys. Rev. B Condens. Matter Mater. Phys. 76 (2007) 1-15, doi:10.1103/PhysRevB.76.054115.

[32] T. Malcherek, M. Fischer, Phase transitions of titanite CaTiSiO5 from density functional perturbation theory, Phys. Rev. Mater. 2 (2018) 1-13, doi:10.1103/PhysRevMaterials.2.023602.

[33] G. Kresse, J. Furthmüller, Efficient iterative schemes for ab initio total-energy calculations using a plane-wave basis set, Phys. Rev. B. 54 (1996) 11169-11186, doi:10.1103/PhysRevB.54.11169.

[34] G. Kresse, D. Joubert, From ultrasoft pseudopotentials to the projector augmented-wave method, Phys. Rev. B. 59 (1999) 1758-1775.

[35] B. Hammer, L.B. Hansen, J.K. Nørskov, Improved adsorption energetics within density-functional theory using revised Perdew-Burke-Ernzerhof functionals, Phys. Rev. B - Condens. Matter Mater. Phys. 59 (1999) 7413-7421, doi:10.1103/PhysRevB.59.7413.

[36] S.L. Dudarev, G.A. Botton, S.Y. Savrasov, C.J. Humphreys, A.P. Sutton, Electronenergy-loss spectra and the structural stability of nickel oxide: an LSDA+U study, Phys. Rev. B. 57 (1998) 1505-1509, doi:10.1103/PhysRevB.57.1505.

[37] J.D. Pack, H.J. Monkhorst, special points for Brillouin-zone integrations, Phys. Rev. B. 16 (1976) 1748-1749, doi:10.1103/PhysRevB.16.1748.

[38] J.D. Pack, H.J. Monkhorst, special points for Brillouin-zone integrations, a reply, Phys. Rev. B. 16 (1977) 1748-1749, doi:10.1103/PhysRevB.16.1748. 
[39] A. Togo, I. Tanaka, First principles phonon calculations in materials science, Scr. Mater. 108 (2015) 1-5, doi:10.1016/j.scriptamat.2015.07.021.

[40] C.R. Robbins, Synthetic CaTiSiO5 and its germanium analogue (CaTiGeO5), Mater. Res. Bull. 3 (1968) 693-698.

[41] J.A. Speer, G.V Gibbs, The crystal structure of synthetic titanite, CaTiOSiO 4, and the domain textures of natural titanites, Am. Mineral. 61 (1976) 238-247.

[42] S. Ghose, Y. Ito, D.M. Hatch, Paraelectric-antiferroelectric phase transition in titanite, CaTiSiO 5, Phys. Chem. Miner. 17 (1991) 591-603.

[43] E. Salje, C. Schmidt, U. Bismayer, Structural phase transition in titanite, CaTiSiO5: a ramanspectroscopic study, Phys. Chem. Miner. 19 (1993) 502-506, doi:10.1007/BF00203191.

[44] M. Kunz, D. Xirouchakis, D.H. Lindsley, D. Hausermann, High-pressure phase transition in titanite (CaTiOSiO4), Am. Mineral 81 (1996) 1527-1530, doi:10.2138/am-1996-11-1225.
[45] M. Kunz, T. Arlt, J. Stolz, In situ powder diffraction study of titanite (CaTiOSiO4) at high pressure and high temperature, Am. Mineral 85 (2000) 1465-1473, doi:10.2138/am-2000-1016.

[46] S. Ghose, Y. Ito, D.M. Hatch, Paraelectric-antiferroelectric phase transition in titanite, CaTiSiO5, Phys. Chem. Miner. 17 (1991) 591-603, doi:10.1007/BF00203838.

[47] K.H. Max Born, Dynamical Theory of Crystal Lattices, (1998).

[48] S.I. Ranganathan, M. Ostoja-Starzewski, Universal elastic anisotropy index, Phys. Rev. Lett. 101 (2008) 3-6, doi:10.1103/PhysRevLett.101.055504.

[49] D. Cherrad, D. Maouche, M. Boudissa, M. Reffas, L. Louail, M. Maamache, K. Haddadi, Y. Medkour, Ultra soft pseudo potential investigation of fundamental physical properties of $\mathrm{CaXO} 3(\mathrm{X}=\mathrm{Sn}$ and $\mathrm{Hf}$ ) distorted perovskites: A reference study to the perfect perovskites, Phys. B Condens. Matter. 429 (2013) 95-105, doi:10.1016/j.physb.2013.08.002. 